\title{
Effect of Magnetic Stimulation in Spinal Cord on Limb Angiogenesis and Implication: A Pilot Study
}

\author{
Dohong Lee, M.D., Jaewon Beom, M.D., Byung-Mo Oh, M.D., Ph.D., Kwan-Sik Seo, M.D., Ph.D.
}

Department of Rehabilitation Medicine, Seoul National University College of Medicine, Seoul 110-744, Korea

\begin{abstract}
Objective To investigate the effect of repetitive magnetic stimulation (rMS) of the spinal cord on limb angiogenesis in healthy rats and explore its implication for the treatment of lymphedema.

Method Twelve adult male Sprague-Dawley rats were divided into four groups as follows: sham rMS followed by tissue harvest 5 minutes later (group 1, n=2), $1 \mathrm{~Hz}$ rMS and tissue harvest 5 minutes later (group 2, n=3), $20 \mathrm{~Hz}$ rMS and tissue harvest 5 minutes later (group 3, n=3), $20 \mathrm{~Hz}$ rMS and tissue harvest 30 minutes later (group 4, $\mathrm{n}=4$ ). Animals were treated with 20 -minute rMS with $120 \%$ of the motor threshold on their left side of upper lumbar spinal cord. Expression of angiogenic factors, that is, Akt, phospho-Akt (pAkt), endothelial nitric oxide synthase (eNOS), phospho-eNOS (p-eNOS) were measured by western blot. Bilateral hindlimb muscles (quadriceps and gastrocnemius) were harvested.

Results Expression of Akt in left quadriceps increased in group 4 compared with group 2 and 3 (3.4 and 5.3-fold each, $\mathrm{p}=0.026$ ). Expression of eNOS in left plus right quadriceps markedly increased in group 3 and 4 compared with group 1 and $2(\mathrm{p}=0.007)$. Expressions of eNOS, Akt and $\mathrm{p}$-eNOS, pAkt in gastrocnemius were not comparable between four groups ( $\mathrm{p}>0.05)$.

Conclusion Repetitive magnetic stimulation of the spinal cord may exert an angiogenic effect closely linked to lymphangiogenesis. It has clinical implication for the possible therapy of lymphedema caused by breast, cervical or endometrial cancer operation. Future studies with the specific lymphatic endothelial cell markers are required to confirm the effect of rMS on lymphangiogenesis.
\end{abstract}

Key Words Magnetic stimulation, Spinal cord, Angiogenesis, Lymphedema, Lymphangiogenesis

Received June 21, 2011; Accepted April 11, 2012

Corresponding author: Kwan-Sik Seo

Department of Rehabilitation Medicine, Seoul National University College of Medicine, 101 Daehak-ro, Jongno-gu, Seoul 110-744, Korea Tel: +82-2-2072-0608, Fax: +82-2-743-7473, E-mail: kurmguro@paran. com

(c) This is an open-access article distributed under the terms of the Creative Commons Attribution Non-Commercial License (http:// creativecommons.org/licenses/by-nc/3.0) which permits unrestricted noncommercial use, distribution, and reproduction in any medium, provided the original work is properly cited.

Copyright () 2012 by Korean Academy of Rehabilitation Medicine

\section{INTRODUCTION}

The lymphatic system plays an important role in the maintaining of the homeostasis of tissue fluids by delivering interstitial fluids rich in proteins to the vascular system via the lymph node and lymphatic channel. ${ }^{1}$ Lymphedema, which is a major complication observed after surgeries such as cervical carcinoma and adnexal tumors, is caused by the anatomical or functional in- 
juries of the lymphatic circulation system. As a result, abnormal edema, the accumulation of interstitial fluid rich in proteins, hyperplasia of fatty tissues, and fibrosis occur. If it becomes chronic, inflammation and infection at the surrounding the edema area repeatedly occur, causing skin fibrosis and extremity deformity. It has been reported that lymphedema occurs in $31-56 \%$ of patients with breast cancer, and $19-24 \%$ of patients with cervical cancer, and that it significantly affect the quality of life..$^{2-5}$

The mechanism and method of lymph node formation has not yet been clearly identified. Various methods have been investigated to change structurally obstructed or retained lymphatic system circulation. Animal studies have been currently conducted to investigate the induction of angiogenesis and lymphangiogenesis of the lower extremities, and factors such as LYVE-1, Proxl, and VEGF have been also investigated..$^{6-8}$ Despite the success of some of these studies, however, most of these studies are experimental so that it is difficult to apply them to clinical practices.

Meanwhile, a recent study was conducted to investigate angiogenesis by applying repetitive magnetic stimulation to the brain tissues of rats. ${ }^{9}$ This study used the principle of the formation of electric field corresponding to magnetic field that is rapidly changed over time. A coil that rapidly changes its magnetic field was positioned closely to the stimulated site, thereby forming an effective electric field and stimulating the neuron. ${ }^{10}$ Unlike to gene transfer that has been introduced for lymphangiogenesis, ${ }^{6,8}$ this magnetic stimulation has been expanding its clinical indications in the human brain neuron as a form of repetitive transcranial magnetic stimulation (rTMS). Various studies have been conducted to investigate the effect of repetitive magnetic stimulation on brain tissues including depression, ${ }^{11}$ migraine, ${ }^{12}$ myofascial pain syndrome, ${ }^{13}$ intractable central pain after stroke ${ }^{14,15}$ and the restoration of motor functions in stroke patients ${ }^{16,17}$ and so on. Studies on the effect of repetitive magnetic stimulation on the brain tissue of rats reported that repetitive magnetic stimulation increased the expressions of eNOS, Akt, and Tie2, genes involved in angiogenesis, in the brain tissues of rats with stroke. ${ }^{9}$

A previous study reported that electric stimulation on the spinal cord facilitated neurogenesis. When the direct current cathode was positioned toward the caudal end, the electrodes attached to the spinal dura, and then elec- tric stimulation was applied, the number of axon was increased at the level in T1 spinal cord injury rats model. ${ }^{18}$ In addition, when $20 \mathrm{~Hz}$ magnetic stimulation was applied to the spinal cord of rats, the antinociceptive effect caused by opioid mechanism was also reported. ${ }^{19}$ A clinical study reported that when magnetic stimulation with a combination of vibration therapy and heat therapy was applied to patients with lower extremity lymphedema, the volume of the injured lower extremities decreased..$^{20}$ However, neither animal experiment nor clinical study has been conducted yet to investigate angiogenesis after the application of magnetic stimulation to the spinal cord.

Accordingly, this preliminary study was performed to investigate angiogenesis closely associated with the lymphangiogenesis of the lower extremities via the observation of the expression of proteins related to angiogenesis by applying repetitive magnetic stimulation to the spinal cord of healthy rats, and to explore the possibility of the application of magnetic stimulation to lymphedema treatment.

\section{MATERIALS AND METHODS}

\section{Subjects}

This study was conducted on 12 8-week-old healthy male Sprague-Dawley rats. The rats were divided into four groups. Group 1 (2 rats) was used as the control group. After standing the coil vertically, $20 \mathrm{~Hz}$ pseudostimulation was applied to the group 1. Five minute later, the rats underwent euthanasia, followed by tissue collection. For group 2 (3 rats), low frequency ( $1 \mathrm{~Hz}$ ) magnetic stimulation was applied, and then tissues were collected 5 minutes later. For group 3 (3 rats), high frequency (20 $\mathrm{Hz}$ ) magnetic stimulation was applied, and then tissues were collected 5 minutes later. For group 4 (4 rats), $20 \mathrm{~Hz}$ magnetic stimulation was applied, and then tissues were collected 30 minutes later.

During the application of repetitive magnetic stimulation, inhalational anesthesia was performed with isofluorane for easier manipulation. Figure-8 coil with a diameter of $25 \mathrm{~mm}$ (Magstim company Ltd, Wales, UK) was used for stimulation.

\section{Methods}

Repetitive magnetic stimulation was applied to the 


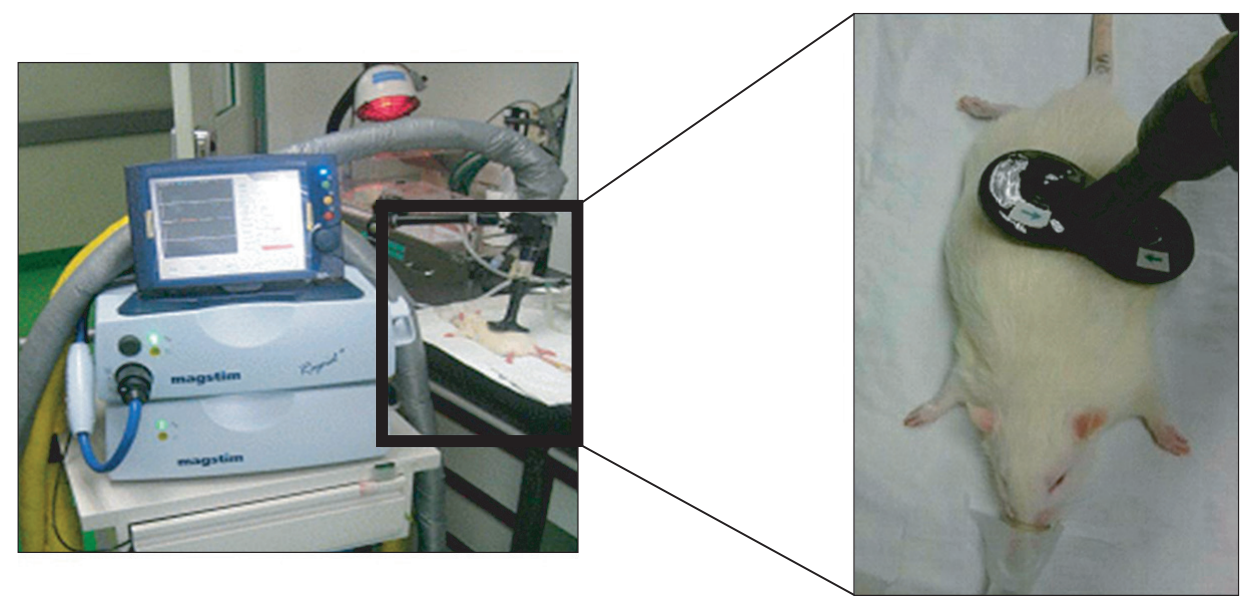

Fig. 1. Repetitive magnetic stimulation on the left side of upper lumbar spinal cord. point of dividing the line that connects the neck to the tail origin of rats in a $2: 1$ ratio, that is, to the upper lumbar. For the left stimulation, the $2: 1$ sectional point was palpated, and then the coil was approximately $45^{\circ}$ inclined to the left, followed by stimulation. To avoid the direct stimulation of the muscle of the lower extremities, the distance between the center of the stimulating coil and the distal thigh muscle was a minimum of $3 \mathrm{~cm}$ apart (Fig. $1)$.

The stimulation intensity was set as $120 \%$ of the motor threshold at which muscle contraction is palpated in the left distal thigh muscle. Stimulation duration was set as $20 \mathrm{~min}$ for both $1 \mathrm{~Hz}$ and $20 \mathrm{~Hz}$ stimulations. For $1 \mathrm{~Hz}$ stimulation, stimulation was applied for $9 \mathrm{sec}$, followed by a $1 \mathrm{sec}$ silent period, a total of 1080 stimulations for 20 min. For $20 \mathrm{~Hz}$ stimulation, stimulation was applied for $2 \mathrm{sec}$, followed by silent period for $28 \mathrm{sec}$, a total of 1600 stimulations for $20 \mathrm{~min}$.

For the expression of proteins related to angiogenesis, endothelial nitric oxide synthase (eNOS) and Akt were measured at the left and right quadriceps femoris muscle, and eNOS, phospho-eNOS (p-eNOS), Akt, and phospho-Akt (pAkt) were analyzed using western blot at the left and right gastrocnemius muscle. Glyceraldehyde 3-phosphate dehydrogenase (GAPDH) was used as an internal control. The result of western blot was quantified using Image J program (NIH, USA).

A statistical analysis was conducted using SPSS 17.0 program for windows. Kruskal-Wallis test was applied for nonparametric procedures, and Mann-Whitney U test was then used to investigate differences in protein expression among the four groups. If $\mathrm{p}<0.05$, it was considered statistically significant.

\section{RESULTS}

No particular adverse event was observed during the period from repetitive magnetic stimulation to euthanasia. The results of repetitive magnetic stimulation on the left upper lumbar are as listed below.

\section{Expressions of eNOS and Akt in the quadriceps femoris muscle}

The Akt expression in the left quadriceps femoris muscle was shown to be 3.4 -fold higher in group 4 than group 2 , and 5.3 -fold higher in group 4 than group $3(\mathrm{p}=0.026)$ (Fig. 2). When the eNOS expression in the left and right quadriceps femoris muscles was summed and analyzed, it was shown to be higher in the groups 3 and 4 than in the groups 1 (control) and 2 ( $\mathrm{p}=0.007$, Kruskal-Wallis test) (Fig. 2). However, no significant difference in summed Akt expression in the left and right quadriceps femoris muscles and eNOS expression in the left quadriceps femoris muscle was found among the four groups (Fig. 2).

\section{Expressions of eNOS and Akt in the gastrocnemius muscle}

No significant difference in the expressions of eNOS and Akt in the left gastrocnemius muscle was found among the four groups. In addition, no significant difference in the expressions of eNOS and Akt in the left and right gastrocnemius muscle was found among the four groups (Fig. 3).

Expressions of p-eNOS and pAkt in the gastrocnemius muscle

No significant difference in the expressions of p-eNOS 
(A)

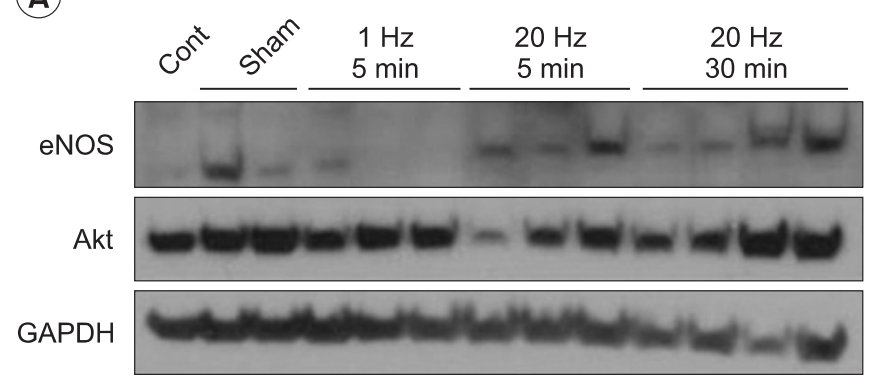

(C)

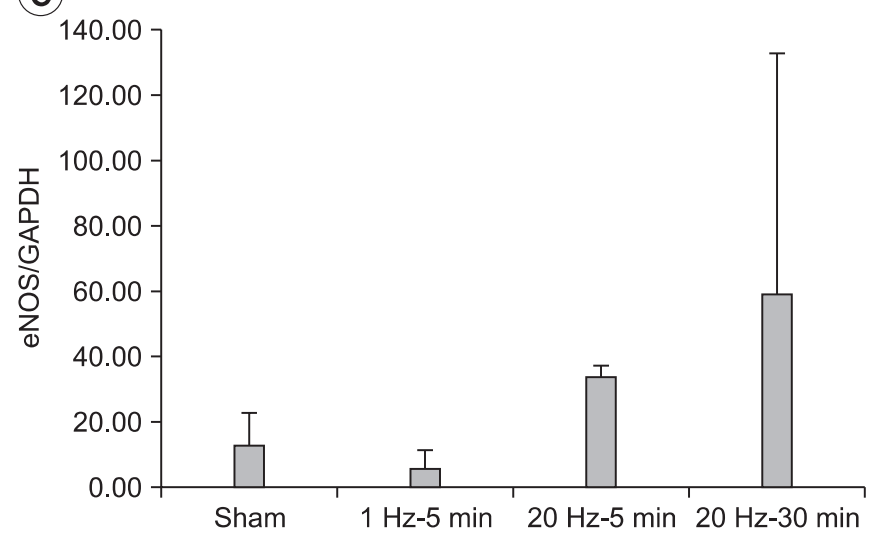

(E)

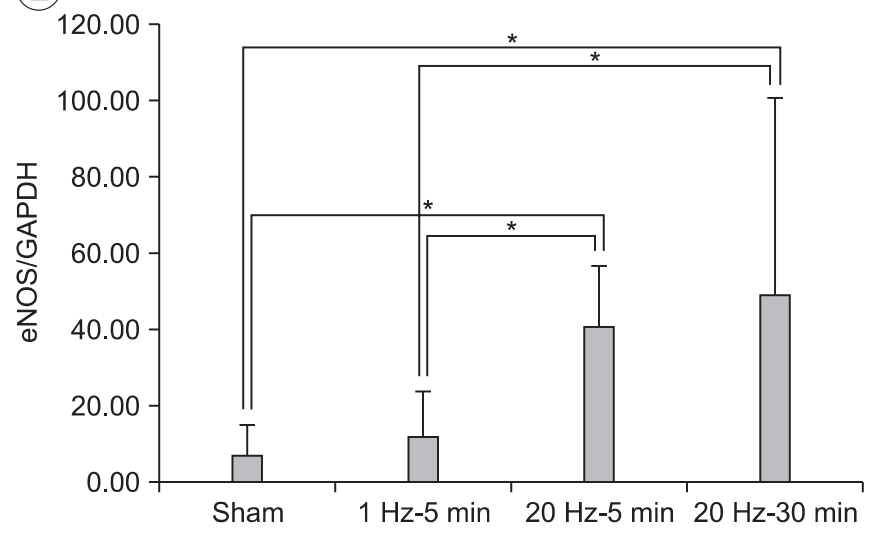

(B)

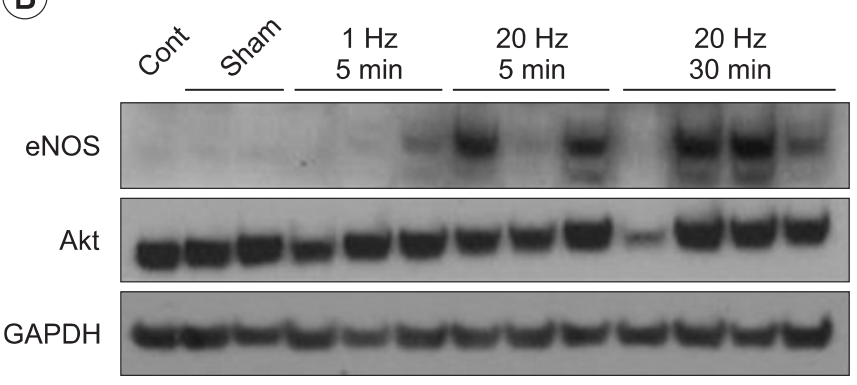

(D)

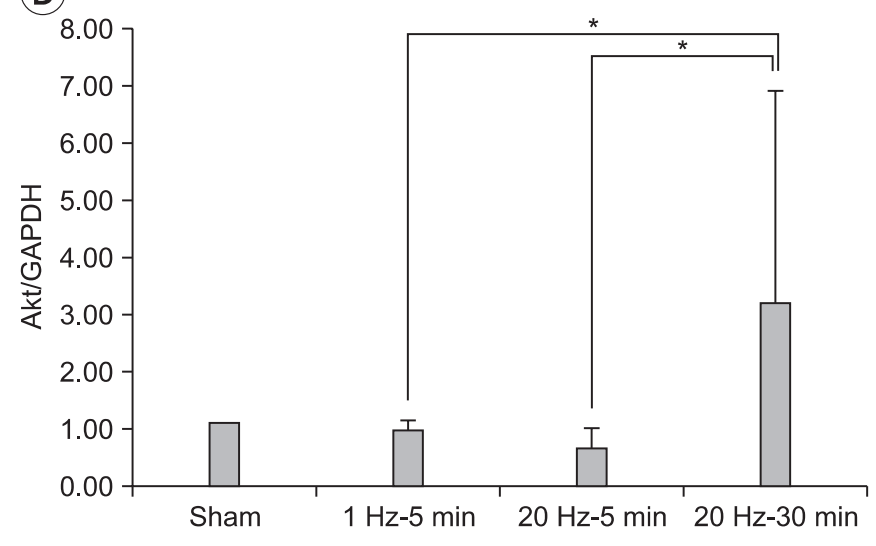

(F)

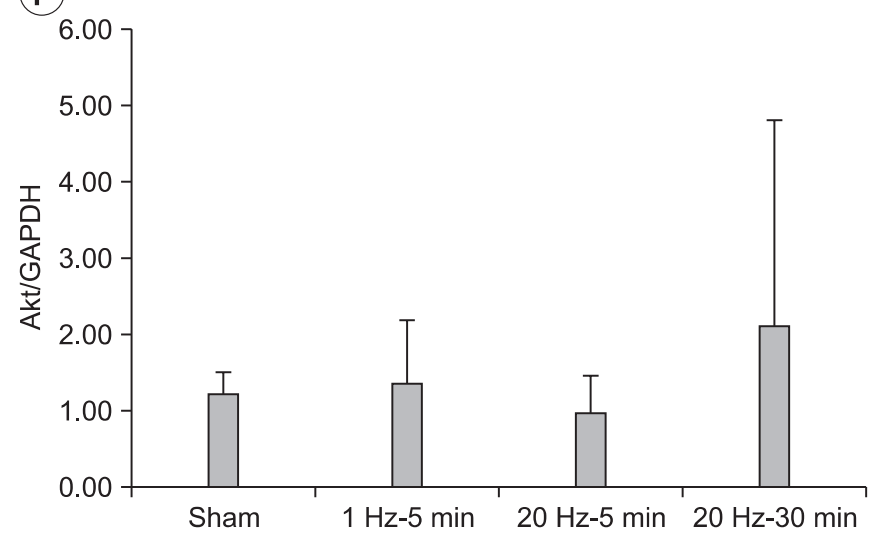

Fig. 2. eNOS and Akt expressions in quadriceps muscle. Western blots of left (A) and right (B) quadriceps, eNOS (C) and Akt (D) expressions in left quadriceps, eNOS (E) and Akt (F) expressions in left+right quadriceps. Expression of Akt in left quadriceps increased in group 4 compared with group 2 and 3 ( 3.4 and 5.3 -fold each, $p=0.026$ ). Expression of eNOS in left+right quadriceps markedly increased in group 3 and 4 (6.4 and 7.7-fold each compared with group 1, 3.5 and 4.2 -fold each compared with group $2, \mathrm{p}=0.007$ ).

and pAkt in the left gastrocnemius muscle was found among the four groups (Fig. 4).

\section{DISCUSSION}

Repetitive transcranial magnetic stimulation (rTMS) can cause changes in cortical excitation that is persistent after stimulation. It is currently being investigated worldwide as a non-invasive cerebral stimulating method, and has been reported to have advantages of less discomfort to patients and acceptable serious adverse events. ${ }^{11}$ rTMS can cause various physiological changes by changing stimulation protocols. It may cause a virtual lesion by transiently halting cortical functions, reduce excitation at 
(A)

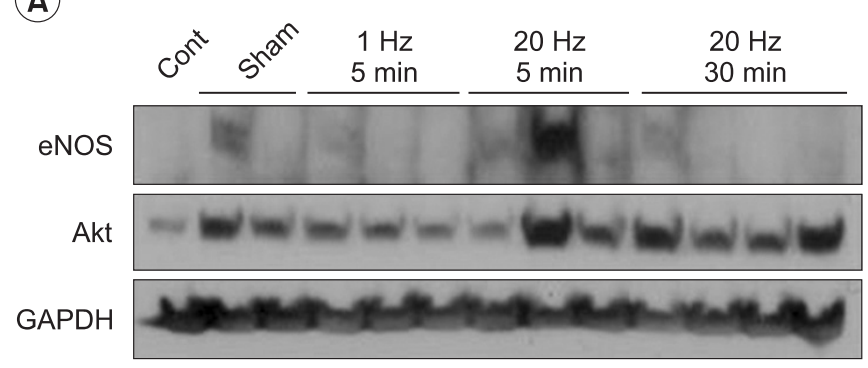

(C)

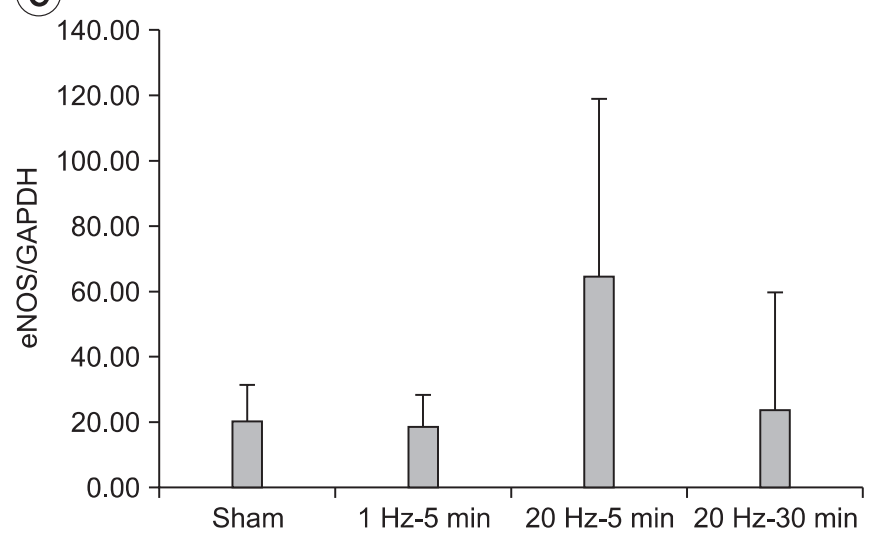

(E)

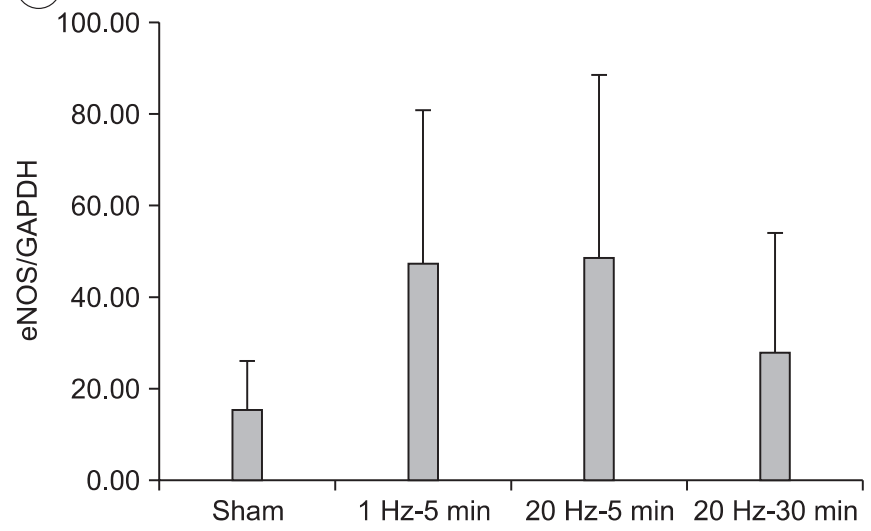

(B)

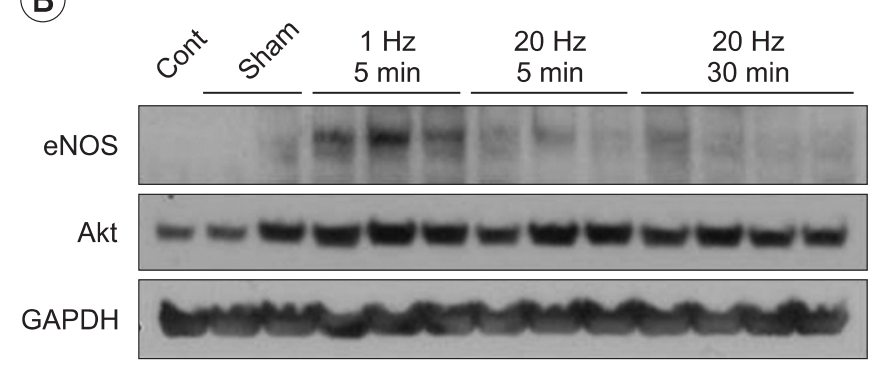

(D)

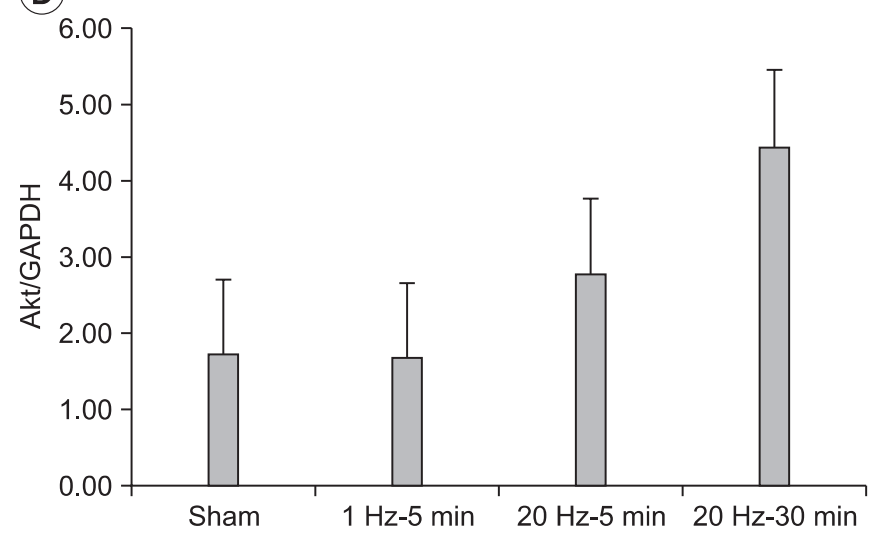

(F)

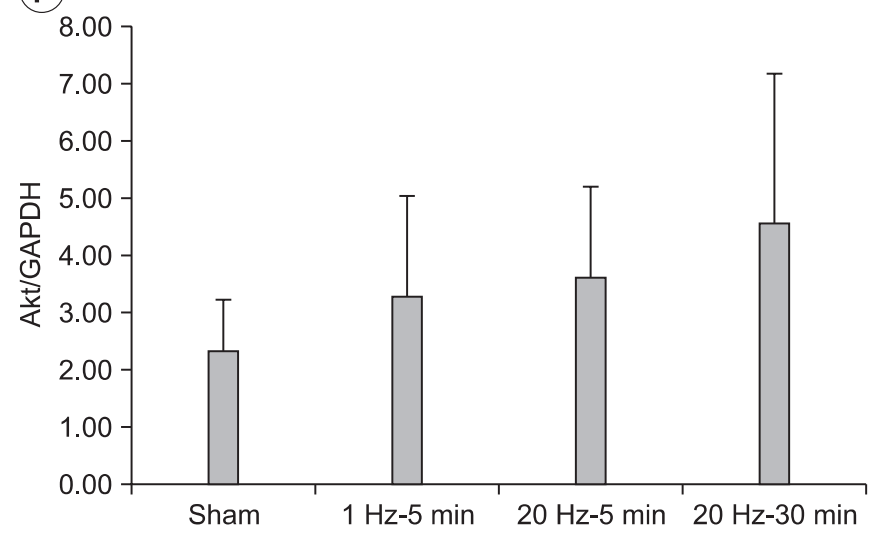

Fig. 3. eNOS and Akt expressions in gastrocnemius muscle. Western blots of left (A) and right (B) gastrocnemius, eNOS (C) and Akt (D) expressions in left gastrocnemius, eNOS (E) and Akt (F) expressions in left+right gastrocnemius. Expressions of eNOS and Akt in gastrocnemius were not comparable between four groups $(p>0.05)$.

a stimulation of low frequency $(1 \mathrm{~Hz})$, and induce activity-dependent plasticity at a stimulation of high frequency (10-20 Hz) by increasing cerebral excitation. In recent studies, a neurovascular coupling mechanism that identifies a correlation between increased local activation of the cerebral neuron and increased cerebral blood flow has been suggested ${ }^{21-23}$ to explain a correlation of repetitive magnetic stimulation with the neuron and various factors related to angiogenesis. ${ }^{9}$
Until now, various methods have been investigated to induce the lymphangiogenesis of the lower extremities via various treatments in animal experiments. When the human hepatocyte growth factor (HGF) gene was transfected to rats with tail lymphedema, the expressions of LYVE-1 and Proxl, lymphatic endothelial cell-specific markers, were shown to have increased, and the lymphedema thickness was shown to have decreased. ${ }^{6}$ In addition, when VEGF-C gene was transfected to rats with 
(A)
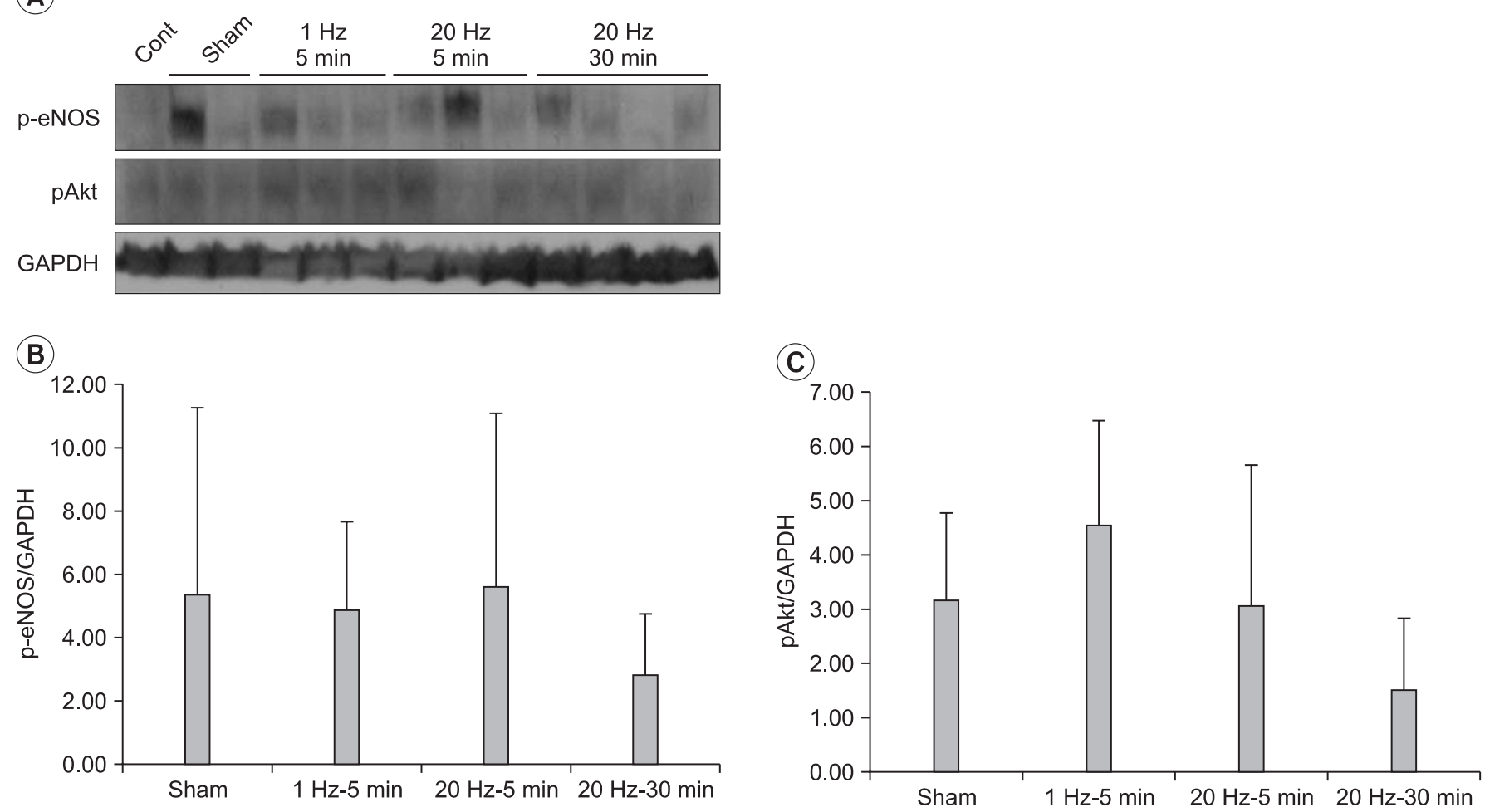

Fig. 4. p-eNOS and pAkt expressions in gastrocnemius muscle. Western blots of left (A) gastrocnemius, p-eNOS (B) and pAkt (C) expressions in left gastrocnemius. Expressions of p-eNOS and pAkt in gastrocnemius were not comparable between four groups ( $\mathrm{p}>0.05)$.

lower extremity lymphedema, and observed via MRI and ultrasonography, the lymphedema thickness was shown to have decreased, and an immunofluorescence analysis showed that lymphangiectasia and lymphangiogenesis increased in the skin, subcutaneous tissue, and muscle layers. ${ }^{8}$ On the other hand, lymphangiogenesis is associated with angiogenesis via VEGF signaling pathway. ${ }^{24,25}$ When electrical stimulation was applied to the lower extremity muscle of rats, angiogenesis, in which VEGF and angiotensin II are involved, was reported to increase. However, no additional data is currently available.

This is the first study that confirmed the effect of repetitive magnetic stimulation applied to the spinal cord on the angiogenesis of the lower extremities. In particular, the increased expressions of eNOS and Akt in the quadriceps femoris muscle indicate angiogenesis. This result is an important clue for possible lymphangiogenesis which is closely associated with angiogenesis. In this study, magnetic stimulation was applied to induce unilateral stimulation by inclining the coil approximately $45^{\circ}$ toward the left lumbar. The expression of eNOS, which is involved in vasodilation and angiogenesis in the left quadriceps femoris muscle, was shown to have increased in the groups 3 and 4, but no significant difference was found. In addition, the expression of Akt, which is involved in cell survival through intracellular signal transduction via growth factors, was shown to be significantly different among the groups for the left quadriceps femoris muscle, but no significant difference was found when the left and right quadriceps femoris muscles are summed. The aforementioned results reflect unilateral stimulation, but bilateral stimulation cannot be ruled out. With respect to local stimulation, Salvador and Miranda ${ }^{26}$ reported that magnetic stimulation using a figure-8 coil with a diameter of $25 \mathrm{~mm}$ ranged within $5 \mathrm{~mm}$. However, as the aforementioned result was obtained from the cerebral tissue, it is unclear if local stimulation on the spinal cord in this study also ranges within $5 \mathrm{~mm}$.

Meanwhile, the summed eNOS expression in the left and right quadriceps femoris muscle was shown to have significantly increased in the groups 3 and 4 . This is likely to attributed to the fact that in addition to direct magnetic stimulation on the spinal cord or nerve root, vasodilation, which was caused by the heat that occurred by local 
heat transmission to the muscles of the lower extremities, occurred at both left and right quadriceps femoris muscles. In fact, it was reported that when repetitive magnetic stimulation was applied to the lateral hand of healthy volunteers for 20 minutes, their skin temperature increased. $^{27}$

The results that the significant differences in the eNOS expression in the bilateral quadriceps femoris muscles and in the Akt expression in the left gastrocnemius muscle were found between the $1 \mathrm{~Hz}$ stimulation groups and $20 \mathrm{~Hz}$ stimulation groups indicate that angiogenesis might vary depending on magnetic stimulation frequency. This is also consistent with the result of a previous study on the cerebral tissues of rats, which was conducted by the authors. ${ }^{9}$ As $20 \mathrm{~Hz}$-high frequency stimulation was shown to have had a stimulating effect on protein expression in the previous study, the tissue was collected 30 minutes after $20 \mathrm{~Hz}$ stimulation, and then analyzed in this study.

It was particularly interesting to mention that compared to the control group, the expressions of p-eNOS and pAkt that are active forms did not increase, but only the expressions of eNOS and Akt increased. If this result is taken into consideration with the fact that the Akt expression in the quadriceps femoris muscle more significantly increased in the group 4 that collected the tissues 30 minutes after the stimulation than in the group 3 that collected the tissues 5 minutes after the stimulation, it is likely that $30 \mathrm{~min}$ after magnetic stimulation is sufficient for the transcription and translation of Akt or eNOS genes. However, the reason for no significant difference in the expressions of $\mathrm{p}$-eNOS and pAkt, active forms via the phosphorylation of Akt and eNOS, is unclear. Thus, in a further study, time sequence should be investigated by measuring the amount of RNA expression to know whether it has been caused by the reduction of protein expression after reaching the peak or by the expression longer than 30 minutes.

This study has a few limitations. First, It is possible that stimulation of the nerve root, not the spinal cord, occurred when magnetic stimulation was applied to the left lumbar. In a previous study, ${ }^{18}$ the electrodes were attached to the spinal dura, and then electric stimulation was applied in a condition of a partial removal of the vertebra. In addition, it is unknown if the spinal cord is stimulated when magnetic stimulation is applied without the resection of the vertebra. Further studies are required to investigate molecular biological changes after transection of the spinal cord.

Second, it cannot be ruled out that angiogenesis is induced by muscle contraction via nerve stimulation. Hudlicka and Price ${ }^{28}$ speculated that hypoxia caused by muscle contraction increases the capillary density of the skeletal muscle. Kanno et al. ${ }^{29}$ reported that electric stimulation that induces muscle contraction stimulated the expression of VEGF protein involved in local angiogenesis. Thus, more studies are also required.

Third, since the degree of angiogenesis was indirectly observed instead of the direct observation of lymphangiogenesis, it is difficult to grant clinical significance in the treatment of lymphedema. In addition, since the lymph vessel is the most substantially distributed in the subcutaneous tissue, it could be different from the vascular distribution of the muscle tissues observed in this study. Thus, further research is required to observe the degree of lymphangiogenesis using lymph vessel-specific markers such as LYVE-1 and Proxl.

Fourth, this is a preliminary study therefore a larger research study is required. It is difficult to collect and analyze tissues located at the deep lumbar area and lower extremities in studies conducted on human beings, an animal experiment is required in a large scale for studying molecular genetic mechanism.

\section{CONCLUSION}

When repetitive magnetic stimulation was applied to the spinal cord of healthy rats, the expression of proteins involved in angiogenesis increased in the lower extremities. This result indicates that angiogenesis could be closely associated with lymphangiogenesis. For the treatment of lymphedema that occurs after surgeries such as breast cancer and uterine cervical carcinoma, a further study is required to investigate the effect of magnetic stimulation applied to the spinal cord on lymphangiogenesis and its mechanism in a molecular level.

\section{REFERENCES}

1. Ohtani O, Ohtani Y. Organization and developmental aspects of lymphatic vessels. Arch Histol Cytol 2008; 71: 1-22 
2. McLaughlin SA, Wright MJ, Morris KT, Sampson MR, Brockway JP, Hurley KE, Riedel ER, Van Zee KJ. Prevalence of lymphedema in women with breast cancer 5 years after sentinel lymph node biopsy or axillary dissection: patient perceptions and precautionary behaviors. J Clin Oncol 2008; 26: 5220-5226

3. Erickson VS, Pearson ML, Ganz PA, Adams J, Kahn KL. Arm edema in breast cancer patients. J Natl Cancer Inst 2001; 93: 96-111

4. Bergmark K, Avall-Lundqvist E, Dickman PW, Henningsohn L, Steineck G. Lymphedema and bladderemptying difficulties after radical hysterectomy for early cervical cancer and among population controls. Int J Gynecol Cancer 2006; 16: 1130-1139

5. Beesley V, Janda M, Eakin E, Obermair A, Battistutta D. Lymphedema after gynecological cancer treatment : prevalence, correlates, and supportive care needs. Cancer 2007; 109: 2607-2614

6. Saito Y, Nakagami H, Morishita R, Takami Y, Kikuchi Y, Hayashi H, Nishikawa T, Tamai K, Azuma N, Sasajima $\mathrm{T}$, et al. Transfection of human hepatocyte growth factor gene ameliorates secondary lymphedema via promotion of lymphangiogenesis. Circulation 2006; 114: 1177-1184

7. Amaral SL, Linderman JR, Morse MM, Greene AS. Angiogenesis induced by electrical stimulation is mediated by angiotensin II and VEGF. Microcirculation 2001; 8: 57-67

8. Liu Y, Fang Y, Dong P, Gao J, Liu R, Hhahbaz M, Bi Y, Ding Z, Tian H, Liu Z. Effect of vascular endothelial growth factor C (VEGF-C) gene transfer in rat model of secondary lymphedema. Vascul Pharmacol 2008; 48: 150-156

9. Oh BM. Low-frequency repetitive transcranial magnetic stimulation in the early subacute phase of stroke enhances the angiogenic mechanisms in rats [dissertation]. Seoul: Seoul National University College of Medicine; 2010

10. Edwards D, Fregni F. Modulating the healthy and affected motor cortex with repetitive transcranial magnetic stimulation in stroke: development of new strategies for neurorehabilitation. NeuroRehabilitation 2008; 23: 3-14

11. O'Reardon JP, Solvason HB, Janicak PG, Sampson $\mathrm{S}$, Isenberg KE, Nahas Z, McDonald WM, Avery D, Fitzgerald PB, Loo C, et al. Reply regarding "efficacy and safety of transcranial magnetic stimulation in the acute treatment of major depression: a multisite randomized controlled trial". Biol Psychiatry 2010; 67: e15-17

12. Brighina F, Giglia G, Scalia S, Francolini M, Palermo A, Fierro B. Facilitatory effects of $1 \mathrm{~Hz}$ rTMS in motor cortex of patients affected by migraine with aura. Exp Brain Res 2005; 161: 34-38

13. Smania N, Corato E, Fiaschi A, Pietropoli P, Aglioti SM, Tinazzi M. Repetitive magnetic stimulation: a novel therapeutic approach for myofascial pain syndrome. J Neurol 2005; 252: 307-314

14. Lefaucheur JP, Drouot X, Menard-Lefaucheur I, Zerah F, Bendib B, Cesaro P, Keravel Y, Nguyen JP. Neurogenic pain relief by repetitive transcranial magnetic cortical stimulation depends on the origin and the site of pain. J Neurol Neurosurg Psychiatry 2004; 75: 612616

15. Hirayama A, Saitoh Y, Kishima H, Shimokawa T, Oshino S, Hirata M, Kato A, Yoshimine T. Reduction of intractable deafferentation pain by navigation-guided repetitive transcranial magnetic stimulation of the primary motor cortex. Pain 2006; 122: 22-27

16. Di Lazzaro V, Pilato F, Dileone M, Profice P, Capone F, Ranieri F, Musumeci G, Cianfoni A, Pasqualetti P, Tonali PA. Modulating cortical excitability in acute stroke: a repetitive TMS study. Clin Neurophysiol 2008; 119: 715-723

17. Mansur CG, Fregni F, Boggio PS, Riberto M, GallucciNeto J, Santos CM, Wagner T, Rigonatti SP, Marcolin MA, Pascual-Leone A. A sham stimulation-controlled trial of rTMS of the unaffected hemisphere in stroke patients. Neurology 2005; 64: 1802-1804

18. Fehlings MG, Tator $\mathrm{CH}$. The effect of direct current field polarity on recovery after acute experimental spinal cord injury. Brain Res 1992; 579: 32-42

19. Lin VW, Hsiao I, Kingery WS. High intensity magnetic stimulation over the lumbosacral spine evokes antinociception in rats. Clin Neurophysiol 2002; 113: 1006-1012

20. Ohkuma M. Treatment of peripheral lymphedema by concomitant application of magnetic fields, vibration and hyperthermia: a preliminary report. Lymphology 2002; 35: 87-90

21. Girouard H, Iadecola C. Neurovascular coupling in the normal brain and in hypertension, stroke, and Al- 
zheimer disease. J Appl Physiol 2006; 100: 328-335

22. Iadecola C. Neurovascular regulation in the normal brain and in Alzheimer's disease. Nat Rev Neurosci 2004; 5: 347-360

23. Allen EA, Pasley BN, Duong T, Freeman RD. Transcranial magnetic stimulation elicits coupled neural and hemodynamic consequences. Science 2007; 317 : 1918-1921

24. Lohela M, Bry M, Tammela T, Alitalo K. VEGFs and receptors involved in angiogenesis versus lymphangiogenesis. Curr Opin Cell Biol 2009; 21: 154-165

25. Benest AV, Harper SJ, Herttuala SY, Alitalo K, Bates DO. VEGF-C induced angiogenesis preferentially occurs at a distance from lymphangiogenesis. Cardiovasc Res 2008; 78: 315-323

26. Salvador R, Miranda PC. Transcranial magnetic stimulation of small animals: a modeling study of the influence of coil geometry, size and orientation. Conf Proc IEEE Eng Med Biol Soc 2009; 2009: 674-677

27. Yambe T, Inoue A, Sekine K, Shiraishi Y, Watanabe M, Yamaguchi T, Shibata M, Maruyama M, Konno S, Nitta S. Effect of the alternative magnetic stimulation on peripheral circulation for regenerative medicine. Biomed Pharmacother 2005; 59 Suppl 1: S174-176

28. Hudlicka O, Price S. The role of blood flow and/or muscle hypoxia in capillary growth in chronically stimulated fast muscles. Pflugers Arch 1990; 417: 6772

29. Kanno S, Oda N, Abe M, Saito S, Hori K, Handa Y, Tabayashi K, Sato Y. Establishment of a simple and practical procedure applicable to therapeutic angiogenesis. Circulation 1999; 99: 2682-2687 